\title{
Production Scheduling USING TWK AND SLK METHODS
}

\author{
Robert Ojstersek, Iztok Palcic \& Borut Buchmeister
}
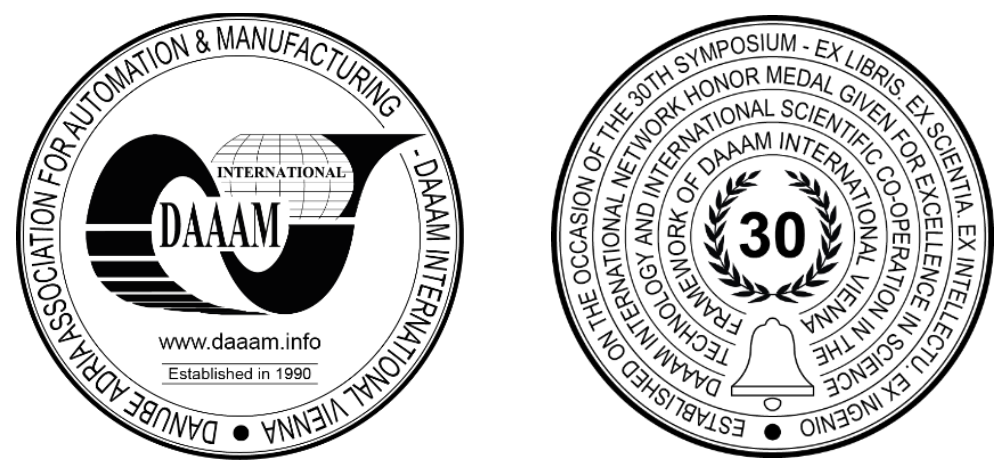

This Publication has to be referred as: Ojstersek, R[obert]; Palcic, I[ztok] \& Buchmeister, B[orut] (2021). Production Scheduling Using TWK and SLK Methods, Proceedings of the 32nd DAAAM International Symposium, pp.0228-0234, B. Katalinic (Ed.), Published by DAAAM International, ISBN 978-3-902734-33-4, ISSN 1726-9679, Vienna, Austria DOI: $10.2507 / 32$ nd.daaam.proceedings.033

\begin{abstract}
The manuscript presents the importance of integrating mathematical methods of due date determination for the maturity optimization in evolutionary computation (EC) methods in the flexible job shop problem (FJSSP). The use of mathematical modelling methods for due date determination, the Slack Method (SLK) for low-dimensional and the Total Work Content Method (TWK) for medium- and high-dimensional problems was used. The results of FJSSP optimization of makespan, machine utilisation and due date scheduling using Multi-Objective Heuristic Kalman Algorithm (MOHKA) algorithm were compared with two existing multi-objective particle swarm algorithms. The obtained results of the tested benchmark datasets proved the high level of FJSSP importance in scheduling. The presented results demonstrate the importance of the due date optimization parameter in achieving a low numbers of tardy jobs, short job tardiness and potentially a lower tardy jobs costs in relation to a short makespan. The main results show that the TWK and SLK method is the key to achieving an on-time and financially justified production system.
\end{abstract}

Keywords: SLK method; TWK method; due date; production scheduling; evolutionary computation

\section{Introduction}

Researchers restricted themselves to optimization parameters that define the due date of jobs [1], assuming independence from other optimization parameters that significantly affect production flexibility [2]. The dynamic changes in FJSSP production due to dynamic customer demand and high-mix low-volume production make Pareto-based optimization approaches a suitable optimization approach [3]. The use of fuzzy approaches that satisfactorily solve the optimization problem of FJSSP production usually treats the problem only at a single level of the primary optimization criteria and restricts the multi-level structure of the FJSSP problem [4]. Heuristic methods, which allow a detailed estimation of the optimization approach and the satisfactory optimization method, are usually limited by the transfer of the optimization results to a real or simulated environment [5]. The need for an efficient optimization method to plan and schedule the FJSSP problem with the optimization parameter of due dates is the key to achieve a comprehensive optimization approach [6]. However, the research results must allow the devaluation of both test and real datasets to adequately integrate the proposed methods into the real production environment [7].

The present research work is organised as follows: In section two, the problem description of the FJSSP is presented through the mathematical formulation of the objective functions and constraints. Section three describes the TWK and SLK methods and proposes a methodology to apply them to the FJSSP. Section four presents the experiments used to evaluate the efficiency and robustness of MOHKA [8] compared to two similar algorithms from EC. Section five presents the optimization results numerically and graphically. The final sixth section describes conclusions and future research steps. 


\section{Problem description}

Flexible job shop production can define flexibility by a four-stage architectural model that treats production flexibility as a multidimensional optimization problem [9]. The degree of production flexibility depends on the characteristics of each production system. Manufacturing systems can define production flexibility in an active or proactive manner [10]. The active mode represents the active use of flexibility to compensate for unknown uncertainties in the production system and deals with both flexibility inside and outside the production process (flexibility of production according to demand and competition policies) [11]. The active approach defines production flexibility as the current ability of the manufacturer to adapt and efficiently use the production system [12]. A proactive approach to production flexibility allows a company to achieve global competitiveness by increasing customer expectations (new orders) and increasing the uncertainty of competing companies [13]. In a proactive approach, production flexibility defines the dynamic change of environmental, social and economic changes [14]. From the point of achieving orders dynamic due dates, it is necessary to adjust production according to customer demand, consider the availability of flexibility of existing products and thus meet customer needs [1]. In the flexible job production optimization problem (FJSSP), the three most common optimization parameters (makespan, total workload of all machine and the workload of the most loaded machine) are defined by Eq. 1, Eq. 2, and Eq. 3 [15]:

- Makespan

$M C=\max \left\{C_{j} \mid j=1, \ldots, n\right\}$,

- total workload of all machine

$T W=\sum_{i=1}^{n} \sum_{j=1}^{n_{i}} \sum_{k=1}^{m} p_{i j k} x_{i j k}, k=1,2, \ldots, m$ and

- the workload of the most loaded machine

$$
M W=\max \sum_{i=1}^{n} \sum_{j=1}^{n_{i}} p_{i j k} x_{i j k}, k=1,2, \ldots, m,
$$

where $C j$ is the completion time of job $j, n$ represents the number of jobs, and $m$ represents the number of machines. These three optimization parameters refer to the time for completing the orders and achieving the highest possible utilization of the machines. In highly flexible job shop production, a very important parameter is the due date of the orders $d j$, which can be very tight to satisfy market demands. Fig. 1 shows the order entry sequence of four jobs, where each job must be executed with a different due date in the production system defined by different machines suitable for perform individual operations. The optimization of the jobs process sequence must be done considering the multi-objective nature of the FJSSP, where three parameters must be minimized (makespan, machine workload and due date).

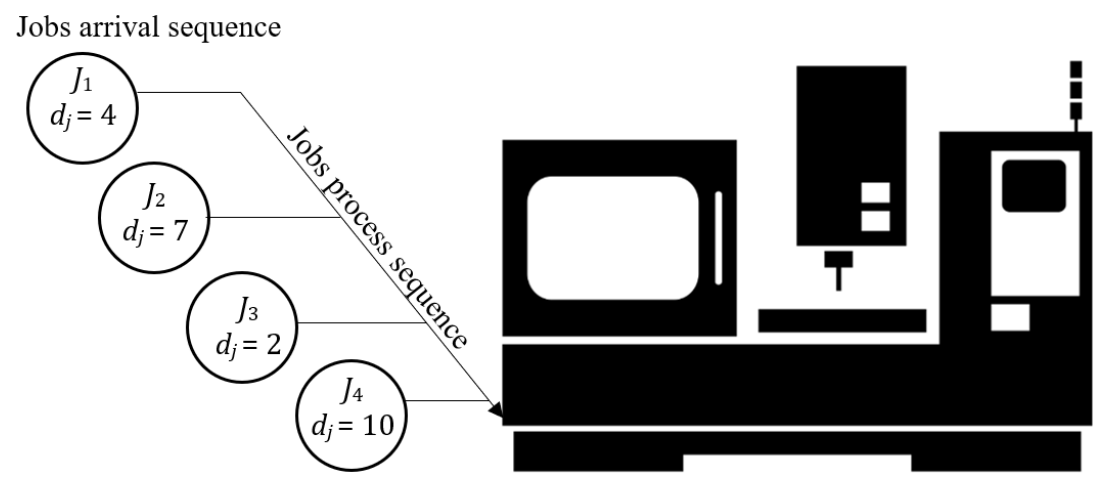

Fig. 1. Jobs arrival sequence

Each job $j$ has a given number of operations $O i$ to be performed on the available machine $m$ from the given set of machines that can perform a single operation. The processing time of the operation $p j k$ varies depending on the assigned machine that can execute the single operation $O i$. The optimization algorithm must arrange the job process sequence in such a way that it handles all four optimization parameters appropriately. For example, using the conventional Earliest Due Date (EDD) priority rule [16], the job process execution sequence would be $J_{3}, J_{1}, J_{2}$, and $J_{4}$. This job process execution sequence handles only one optimization parameter $d j$, which is defined as follows: the due date of job $j$ is the estimated dispatch date of job $j$ (dispatch date promised to the customer or scheduled in the company). Completing order $j$ after the due date is allowed but represents an additional financial or time penalty to the company. When considering the optimization parameter $d j$, important associated goals must be established. The primary objective is to reduce the maximum lateness, which is defined as in Eq. 4: 
$L_{\max }=\max \left(L_{j}, \ldots, L_{n}\right)$,

where the lateness of an individual job $j$ is defined by the Eq. 5:

$$
L_{j}=C_{j}-d_{j}
$$

depends on the completion time of job $j$ and the assumed delivery time of job $\mathrm{dj}$. The temporal $L_{\max }$ is easier to define with the parameter number of tardy jobs. This optimization parameter only defines whether the individual job $j$ has exceeded the estimated delivery time or not. Tardiness of job $j$ is defined as in Eq. 6:

$$
T_{j}=\max \left(C_{j}-d_{j}, 0\right),
$$

and the corresponding target function defined by Eq. 7 .

$$
\sum_{j=1}^{n} T j .
$$

Due to the shortcomings of the above optimization function, which relates to some very tardy jobs, it is useful to determine the importance weights of jobs $j$ by $w_{j}$ parameter. The higher the weight, the more important the job $j$ is. The assumption in this research work is that the given weight $w_{j}$ refers only to the importance of a single job $j$, which can be weighted directly by the scheduling team of the manufacturing system. The importance of multicriteria decision making between the four optimization parameters (MC, TW, MW and dj ) does not determine the importance of the correlation between them.

\section{TWK and SLK methods formulation}

To model the due dates of jobs, a random FJSSP data sets are generated and divided into three groups in terms of their complexity dimensions [17]:

- Low dimensional optimization problems, the dataset of J5, M11, O66 has been configured to evaluate the capabilities of MOHKA with respect to the optimization results of conventional priority rules.

- Middle dimensional optimization problem is represented by the dataset of J10, M11, O122.

- High dimensional optimization problem is represented by dataset of J15, M11, O176.

TWK [18] method due date optimization parameter modelling is performed according to the Eq. 8.

$$
\boldsymbol{d}_{j}=a t_{j}+K_{x} * \sum_{i \in o_{j}} p_{i, j, k}
$$

The tightness allowance factor of the due date $K_{x}$ determines the tightness of individual orders. Numerical values in the literature, range it as $3 \leq K_{x} \leq 5$, for the TWK method. The smaller the value of the tightness allowance factor, the tighter the due date of order $j$ is. The experiments in the manuscript use the value of $K_{x}=3$. The due date modelled by the TWK method depends on the arrival time of order $j\left(a_{t j}\right)$, the total time of processing all operations $\left(p_{i j k}\right)$, and the described tightness allowance factor. The MOHKA algorithm schedules the orders according to four optimization criteria, including the due date $d j$. The comparison of the adequacy of the proposed MOHKA method is performed using two comparison algorithms: Multi-objective particle swarm optimization (MOPSO) [19] and Bare-bones multi-objective particle swarm optimization (BBMOPSO) [20]. These two algorithms do not use an integrated mathematical decision model to schedule jobs according to the due date criterion, this criterion is numerically calculated in the experiment at the end of the optimization results. As stated in the initial research question, the due date parameter in the FJSSP optimization problem is not well studied, especially when it comes to using the EC method to obtain optimal solutions. All the algorithms in the experiment use the same initialization parameters: Population size $\left(N_{s}=300\right)$, maximum number of archived nondominant solutions $\left(N_{a}=100\right)$, and maximum number of algorithm iterations (MaxIter $=300$ ).

The optimization parameter for scheduling jobs by due date is analysed using three criteria: Number of tardy jobs, average job tardiness and tardy jobs cost. The tardy jobs costs is modelled as shown in Eq. 9, where the initial job cost $\left(J_{\text {cost }}\right)$ is multiplied by the constant value of three, divided by the value constant $K_{s}$, and multiplied by subtracting the completion time $C_{j}$ and the due date $d_{j}$.

$$
L_{\text {cost }}=\left\{\begin{array}{l}
\frac{3 * J_{\text {cost }}}{K_{S}} *\left(C_{j}-d_{j}\right), \text { if } C_{j}>d_{j} \\
0, \text { if } C_{j} \leq d_{j}
\end{array}\right.
$$

A constant numerical value determines three times the cost of tardy jobs compared to the cost of orders completed on time (presenting penalty costs). The value $K_{S}$ is automatically determined time variable, presenting individual order makespan. The parameter $J_{\text {cost }}$ is the machines cost processing an individual order on a specific machine.

The modelling of the due dates and the achievement of the other three optimization parameters were performed using conventional methods (priority rules) and the heuristic GSBR (General Shifting Bottleneck Routine) method to evaluate the efficiency of the conventional scheduling methods compared to the proposed MOHKA EC method. The comparison 
of priority rules and GSBR method is performed in the Lekin software environment. Since the Lekin software environment only allows the optimization of datasets with up to one hundred operations in the FJSSP optimization problem, the evaluation is performed with a randomly generated benchmark dataset classified as a low-dimensional optimization problem $J_{5}, M_{11}, O_{66}$. A randomly generated benchmark dataset does not contain data where two or more operations are performed on the same machine within a single job. This limitation is made according to the Lekin decision making strategies. In contrast to larger benchmark datasets where the TWK method is used to model due dates, the SLK (Slack) [21] method is recommended for smaller datasets. For low-dimensional dataset and SLK method, the due date parameter is modelled by the Eq. 10 .

$$
d_{j}=a t_{j}+\sum_{i \in o_{j}} p_{i, j, k}+K_{y}
$$

In the SLK method the determination of the time reserve constant $K_{y}$ determines the looseness-tightness of due dates. Reserve constant values given by the literature are $4 \leq K_{y} \leq 16$. In the presented research work the middle value of $K_{y}=$ 8 is used.

\section{Experiment design}

To test the performance of the MOHKA algorithm for job scheduling (according to the due date parameter) four randomly generated benchmark datasets were used, describing FJSSP optimization problem. The datasets were randomly created using the dependency function between different optimization parameters. We divided these benchmark datasets into three groups according to the complexity of the optimization problem (low-, middle- and high-dimensional). Due to the classification of the used datasets into different groups according to their complexity we have been able to evaluate individual benchmark and algorithm capabilities. Use of TWK and SLK method for different optimization problems were made as: TWK method for medium and high dimensional optimization problems and SLK method for low dimensional optimization problems. With the presented classification approach, the complexity of the optimization problems can be evaluated more accurately to determine the advantages and limitations of each method. The proposed MOHKA algorithm performed the optimization of data sets with four parameters as described in section three: makespan (MC), total workload of all machines (TW), maximum workload of a single machine (MW) and added due date parameter $(d j)$. The obtained optimization results were compared with two particle swarm based multi-objective optimization algorithms (MOPSO and BBMOPSO). The experiments were performed on a personal computer with Intel i7 processor and 16 GB internal memory.

\section{Due date scheduling results}

The results in Table 1 show the high reliability of scheduling jobs with the TWK method considering due dates with the MOHKA optimization algorithm. The method is successful in scheduling jobs with tight due dates, low average jobs tardiness, potentially low tardy jobs cost and short orders makespan. The medium sized data set $J_{10}, M_{11}, O_{122}$ did not cause any problems for all three evolutionary computation algorithms in scheduling orders with tight due dates of the TWK method with the tightness coefficient of $K_{x}=3$. No job has missed the scheduled due date, which in turn resulted in no additional tardy jobs costs in the production system. Since only the referential MOHKA optimization algorithm considers the mathematical architecture of the TWK method, we see that the results of multi-objective optimization have a positive impact on the achievement of the minimum orders makespan. The MOHKA algorithm achieves the shortest makespan with up to $226 \mathrm{~h}$, in contrast to MOPSO and BBMOPSO, where the makespan is $272 \mathrm{~h}$ and $247 \mathrm{~h}$, respectively.

When the dimension of the datasets increases from medium to high dimensional optimization problems, the difference between the optimization results of the comparison and reference algorithms becomes more obvious, as shown in Fig. 2 (green graph lines). In dataset $J_{15}, M_{11}, O_{176}$, the MOHKA algorithm terminates orders such that four orders are late by the expected due date, with an average job tardiness of $22.8 \mathrm{~h}$, resulting in a tardy jobs costs of 2,738.6 EUR. The MOPSO algorithm finishes orders such that only two orders miss the expected due date, but with higher average job tardiness of $54.6 \mathrm{~h}$, which is $139 \%$ higher average job tardiness than the MOHKA algorithm. Longer average job tardiness leads to higher tardy jobs costs, which is 3,559.8 EUR for the MOPSO algorithm. The BBMOPSO algorithm had the most inefficient job schedule, six orders missing the scheduled due date, with an average job tardiness of $24.72 \mathrm{~h}$ and a high tardy jobs costs of 4,270.2 EUR. With that results BBMOPSO represents a 56.6\% increase in the tardy jobs cost compared to the MOHKA algorithm. The results show that the MOHKA algorithm is most successful even with an order makespan parameter of $347 \mathrm{~h}$, which is shorter than $407 \mathrm{~h}$ for the BBMOPSO algorithm and 356 for the MOPSO algorithm. Based on the above optimization results of the due date FJSSP optimization, the complexity of solving higher dimensional optimization problems with the TWK and SLK methods increases. The initial research question is confirmed for the highdimensional dataset of $J_{20}, M_{11}, O_{240}$, where the reference MOHKA algorithm dominates over the results of the two comparison algorithms shown in Fig. 2 (blue graph lines). The reference MOHKA algorithm has the number of tardy jobs equal to eight, with an average job tardiness of $37.32 \mathrm{~h}$ compared to $53.28 \mathrm{~h}$ and $56.16 \mathrm{~h}$ for MOPSO and BBMOPSO, respectively, which corresponds the higher number of tardy jobs of twelve for MOPSO and twenty two for BBMOPSO. Given the higher number of tardy jobs and the longer average job tardiness, the cost of tardy jobs is also higher for the two comparison algorithms is also higher than for the reference algorithm MOHKA, amounting to 7,318.1 EUR. 


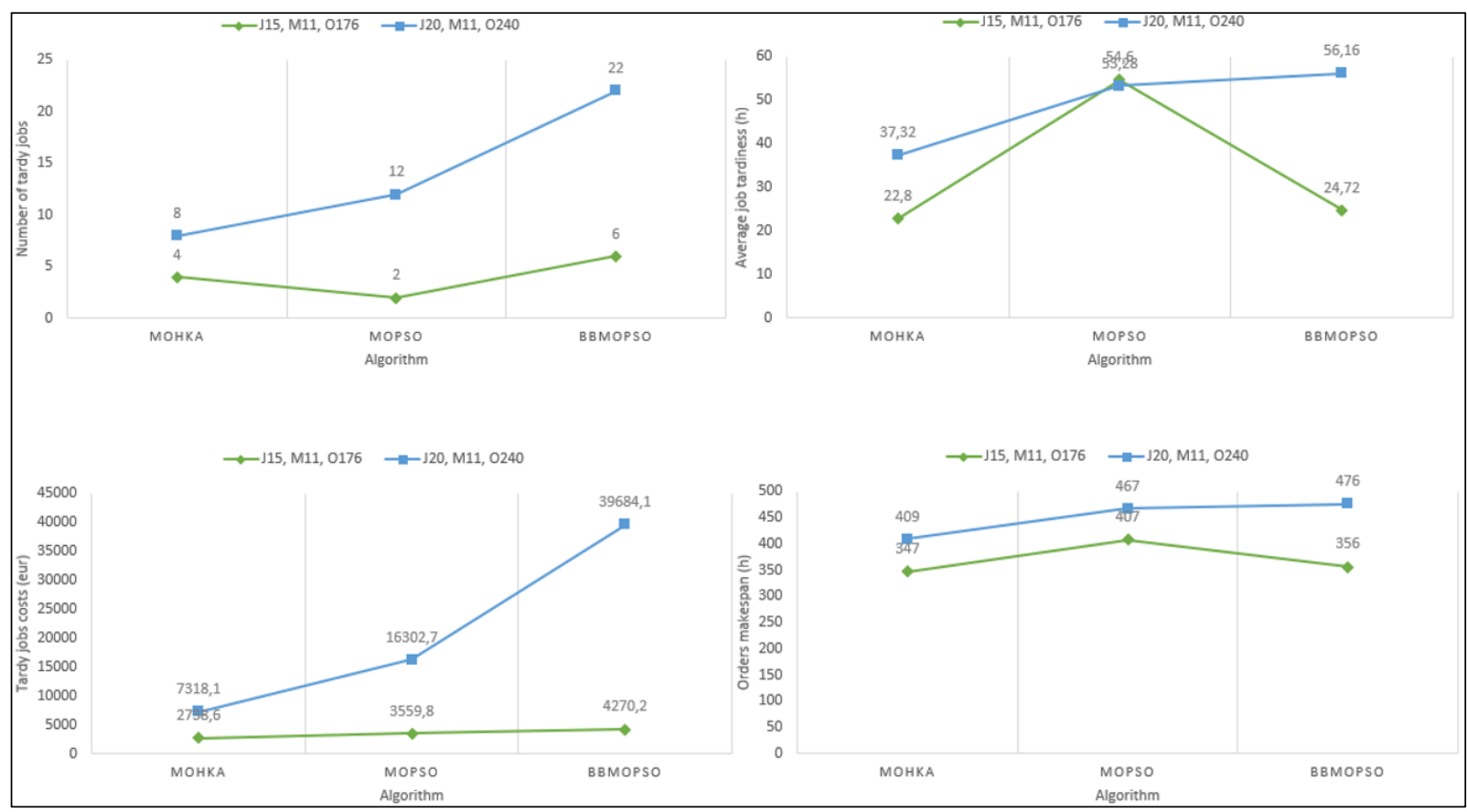

Fig. 2. Optimization results of datasets $J_{15}, M_{11}, O_{176}$ and $J_{20}, M_{11}, O_{240}$.

\begin{tabular}{|c|c|c|c|c|c|}
\hline Algorithm & Dataset & $\begin{array}{c}\text { Number of } \\
\text { tardy jobs }\end{array}$ & $\begin{array}{c}\text { Average job } \\
\text { tardiness (h) }\end{array}$ & $\begin{array}{c}\text { Tardy jobs costs } \\
\text { (EUR) }\end{array}$ & $\begin{array}{c}\text { Orders } \\
\text { makespan (h) }\end{array}$ \\
\hline \multirow{3}{*}{ MOHKA } & $J_{10}, M_{11}, O_{122}$ & 0 & 0 & 0 & 226 \\
\cline { 2 - 6 } & $J_{15}, M_{11}, O_{176}$ & 4 & 22.8 & $2,738.6$ & 347 \\
\cline { 2 - 6 } & $J_{20}, M_{11}, O_{240}$ & 8 & 37.32 & $7,318.1$ & 409 \\
\hline \multirow{3}{*}{ MOPSO } & $J_{10}, M_{11}, O_{122}$ & 0 & 0 & 0 & 272 \\
\cline { 2 - 6 } & $J_{15}, M_{11}, O_{176}$ & 2 & 54.6 & $3,559.8$ & 407 \\
\cline { 2 - 6 } & $J_{20}, M_{11}, O_{240}$ & 12 & 53.28 & $16,302.7$ & 467 \\
\hline \multirow{3}{*}{ BBMOPSO } & $J_{10}, M_{11}, O_{122}$ & 0 & 0 & 0 & 247 \\
\cline { 2 - 6 } & $J_{15}, M_{11}, O_{176}$ & 6 & 24.72 & $4,270.2$ & 356 \\
\cline { 2 - 6 } & $J_{20}, M_{11}, O_{240}$ & 22 & 56.16 & $39,684.1$ & 476 \\
\hline
\end{tabular}

Table 1. Algorithm's optimization results

With the MOPSO algorithm, the tardy jobs costs amount to 16,302.7 EUR, while with BBMOPSO it is 39,684.1 EUR. The presented results prove the high importance of mathematical modelling with the parameters of due date optimization, as they have a crucial impact on the makespan and financial justification of highly dynamic manufacturing systems [22]. A suitable mathematical model of the multi-objective optimization problem is also reflected in the achievement of a short order makespan. For the high-dimensional data set of $J_{20}, M_{11}, O_{240}$, the reference MOHKA algorithm achieved a makespan of $409 \mathrm{~h}$ and the two comparison algorithms $467 \mathrm{~h}$ and $476 \mathrm{~h}$, respectively.

\subsection{Results of the mathematical modelling with SLK method}

With the aim of comparing the solutions of MOHKA algorithm and the solutions of conventional priority rules, a comparison of the results of MOHKA optimization with the results of job scheduling of $J_{5}, M_{11}, O_{66}$ in the Lekin software environment for the low-dimensional optimization problem was performed. Table 2 shows the optimization results of a $J_{5}, M_{11}, O_{66}$ data set and comparisons between MOHKA and priority rules optimization results, using SLK method. The optimization was performed using MOHKA optimization algorithm in MATLAB software environment and seven optimization approaches in Lekin software environment. Of the seven optimization approaches, six are conventional priority rules and one is a heuristic algorithm called General Shifting Bottleneck Routine (GSBR). The SLK method with a time reserve constant of $K_{y}=8$ was used to optimise the due dates. The results show high reliability of scheduling production jobs scheduling with MOHKA optimization algorithm. In the considered dataset, MOHKA schedules orders in such a way that two orders miss the scheduled due dates with an average job tardiness of $11.4 \mathrm{~h}$ and a tardy jobs costs of 3,181 EUR. Among the six priority rules, we see that the five priority rules, except for the priority rule SPT, terminate orders in a way that all five orders miss the scheduled due date. The average job tardiness is higher than the MOHKA algorithm, ranging from $35.3 \mathrm{~h}$ for the priority rule CR to $57.6 \mathrm{~h}$ for the priority rule LPT. Moreover, the cost of delayed orders is significantly higher. The only algorithm that partially approximates the results of the MOHKA algorithm is the heuristic GSBR algorithm, where four orders are tardy with an average job tardiness of 11.3. Another significant 
difference is that the MOHKA algorithm finishes jobs so that they are completed in a makespan of $118.8 \mathrm{~h}$, while all other algorithms finish jobs with makespan between $249.6 \mathrm{~h}$, by GSBR algorithm, and $307.2 \mathrm{~h}$ by CR priority rule.

\begin{tabular}{|c|c|c|c|c|c|c|c|c|}
\hline Algorithm & MOHKA & EDD & MS & FCFS & LPT & SPT & CR & GSBR \\
\hline Number of tardy jobs & 2 & 5 & 5 & 5 & 5 & 4 & 5 & 4 \\
\hline Average job tardiness (h) & 11.4 & 41.8 & 57.1 & 49.9 & 57.6 & 38.9 & 35.3 & 11.3 \\
\hline Tardy jobs costs (EUR) & 3,181 & 13,590 & 17,618 & 15,984 & 17,323 & 12,684 & 10,686 & 4,447 \\
\hline Orders makespan (h) & 118.8 & 253.2 & 265.2 & 258 & 260.4 & 279.6 & 307.2 & 249.6 \\
\hline
\end{tabular}

Table 2. SLK optimization results of MOHKA algorithm vs. priority rules due date scheduling

The presented optimization results prove the high term fidelity of the MOHKA algorithm to achieve tight due dates from low-dimensional optimization cases (with SLK method) up to medium and high-dimensional optimization cases (with TWK method) in comparison to optimization solutions according to MOSPO, BBMOPSO and priority rules.

\section{Conclusion}

Scheduling multi-objective FJSSP optimization problem is defined as a non-deterministic polynomial-time hard optimization problem. The original research question of scheduling FJSSP production with the optimization parameter of due date importance and considering the standard optimization parameters related to the makespan of orders and machine utilisation was evaluated in the presented research using MOHKA optimization method and SLK and TWK methods for modelling due dates. As the number of optimization parameters increases, the computational complexity of the optimization algorithm increases. The presented research presents the integration of the mathematical structure of SLK (for low-dimensional optimization problems) and TWK method (for medium- and high-dimensional optimization problems) into the MOHKA optimization algorithm. The proposed MOHKA algorithm was used to schedule benchmark data sets, focusing on achieving an order due date, corelated with due date tightness coefficient. The optimization results were compared with the results of two EC algorithms: MOPSO and BBMOPSO optimization algorithms. The disadvantage of the comparative optimization methods (MOPSO and BBMOPSO) becomes obvious when dealing with medium and high dimensional optimization problems in scheduling FJSSP. The limited scheduling capabilities of the MOPSO and BBMOPSO algorithms are reflected in the limited mathematical structure, considering due date, which do not consider the SLK and TWK methods as decision parameters in obtaining optimally scheduled orders. The optimization results of the reference MOHKA algorithm prove the high importance of the due date optimization parameter, since the proposed method optimises the order scheduling with respect to the two comparison algorithms for low, medium, and high dimensional optimization problems. Since it involves multi-objective decision making and finding trade-offs between different (even conflicting) optimization parameters. The results of MOHKA algorithm prove its high ability to achieve all four optimization parameters (MC, TW, MW and $d_{j}$ ) equally and efficiently. The short order makespan, tight due dates, low average order tardiness and associated low associated job tardiness costs are achieved. The answer to the question about the efficiency of EC methods in multicriteria decision making compared to the conventional optimization approach of priority rules was given by the presented study, in which the optimization results of MOHKA algorithm are compared with the optimization results of six priority rules and an integrated heuristic method in the Lekin software environment. The obtained results demonstrate the high dominance of the optimization results of the EC method MOHKA, which completes the FJSSP production most efficiently according to the used low-dimensional dataset for all optimization parameters. Randomly generated benchmark datasets were the basis for conducting the validation of the applicability of the proposed method in real manufacturing systems, and the satisfactory optimization results were demonstrated in the experiment. Since the presented research work only deals with FJSSP, which is the main part of the research problem of multi-objective optimization of job shop production, it is necessary to further investigate the importance of scheduling in dynamic job shop production (DJSSP). The main features are dynamic job changes during the execution of the algorithm (in the initialization phase, the entire job record is unknown), machine failures during the execution of operations, and determining the importance of different jobs characteristics to be studied. Further research on the DJSSP research problem would remove the limitations of current research, in which the FJSSP optimization problem assumes of an initially known order data set, an initially empty production system, uniform importance of orders, and known production capacities that do not change during operations. Further jobs shop due date scheduling usability can be transferred to mass production and other types of manufacturing systems.

\section{Acknowledgments}

This research was funded by the Slovenian Research Agency (ARRS), Research Core Grant P2-0190.

\section{References}

[1] Saha, C.; Aqlan, F.; Lam, S. S. \& Boldrin, W. (2016). A decision support system for real-time order management in a heterogeneous production environment, Expert Systems with Applications, Vol. 60, 16-26, doi:10.1016/j.eswa.2016.04.035 
[2] Ojstersek, R. \& Buchmeister, B. (2020). The impact of manufacturing flexibility and multi-criteria optimization on the sustainability of manufacturing systems, Symmetry, Vol. 12, No. 1, Paper 157, 22 pages, doi:10.3390/sym12010157

[3] Wisittipanich, W. (2013). An efficient pso algorithm for finding pareto-frontier in multi-objective job shop scheduling problems, Vol. 12, No. 2, 151-160, 10.7232/iems.2013.12.2.151

[4] Koblasa, F.; Kraliková, R. \& Votrubec, R. (2020). Influence of EA control parameters to optimization process of FJSSP problem, International Journal of Simulation Modelling, Vol. 19, No. 3, 387-398, doi:10.2507/ijsimm19-3519

[5] Ojstersek, R.; Palcic, I.; Buchmeister, B. (2020). Simulation modelling of environmental and economic aspects in manufacturing systems, Proceedings of 31st DAAAM International Symposium on Inteligent Manufacturing and Automation, Katalinic, B. (Ed.), pp. 436-443, DAAAM International, Zadar, doi: 10.2507/31st.daaam.proceedings.061

[6] Xu, Y.; Wang, L.; Wang, S. \& Liu, M. (2013). An effective shuffled frog-leaping algorithm for solving the hybrid flow-shop scheduling problem with identical parallel machines, Engineering Optimization, Vol. 45, No. 12, 14091430, doi:10.1080/0305215X.2012.737784

[7] Ojstersek, R.; Lalic, D. \& Buchmeister, B. (2019). A new method for mathematical and simulation modelling interactivity: A case study in flexible job shop scheduling, Advances in Production Engineering And Management, Vol. 14, No. 4, doi:10.14743/apem2019.4.339

[8] Ojstersek, R.; Zhang, H.; Shifeng, L. \& Buchmeister, B. (2018). Improved heuristic kalman algorithm for solving multi-objective flexible job shop scheduling problem, Procedia Manufacturing, Vol. 17, 985-902, doi:10.1016/j.promfg.2018.10.142

[9] Ripon, K. S. N.; Siddique, N. H. \& Torresen, J. (2011). Improved precedence preservation crossover for multiobjective job shop scheduling problem, Evolving Systems, Vol. 2, No. 2, 119-129, doi:10.1007/s12530-010-9022$\mathrm{x}$

[10] Yang, W. \& Takakuwa, S. (2017). Simulation-based dynamic shop floor scheduling for a flexible manufacturing system in the industry 4.0 environment, 2017 Winter Simulation Conference, pp. 3908-3916, IEEE, Las Vegas, doi:10.1109/WSC.2017.8248101

[11] Istokovic, D.; Perinic, M.; Vlatkovic, M. \& Brezocnik, M. (2020). Minimizing total production cost in a hybrid flow shop: a simulation-optimization approach, International Journal of Simulation Modelling, Vol. 19, No. 4, 559-570, doi:10.2507/ijsimm19-4-525

[12] Ojstersek, R. \& Buchmeister, B. (2017). Use of simulation software environments for the purpose of production optimization, Proceedings of the 28th DAAAM International Symposium, pp. 750-758, B. Katalinic (Ed.), DAAAM International, Zadar, doi:10.2507/28th.daaam.proceedings.106

[13] Zhang, H.; Buchmeister, B.; Li, X. \& Ojstersek, R. (2021). Advanced metaheuristic method for decision-making in a dynamic job shop scheduling environment, Mathematics, Vol. 9, No. 8, Paper 909, 22 pages, doi:10.3390/math9080909

[14] Frutos, M.; Tohmé, F.; Delbianco, F. \& Miguel, F. (2016). An alternative hybrid evolutionary technique focused on allocating machines and sequencing operations, International Journal of Industrial Engineering Computations, Vol. 7, No. 4, 585-596, doi:10.5267/j.ijiec.2016.4.002

[15] Xiang, W.; Yin, J. \& Lim, G. (2015). An ant colony optimization approach for solving an operating room surgery scheduling problem, Computers \& Industrial Engineering, Vol. 85, 335-345, doi:https://doi.org/10.1016/j.cie.2015.04.010

[16] Hao, X.; Gen, M.; Lin, L. \& Suer, G. A. (2017). Effective multiobjective EDA for bi-criteria stochastic job-shop scheduling problem, Journal of Intelligent Manufacturing, Vol. 28, No. 3, 833-845, doi:10.1007/s10845-014-10260

[17] Ojstersek, R.; Tang, M. \& Buchmeister, B. (2020). Due date optimization in multi-objective scheduling of flexible job shop production, Advances in Production Engineering \& Management, Vol. 15, No. 4, 481-492, doi:10.14743/apem2020.4.380

[18] Huang, J. \& Süer, G. A. (2015). A dispatching rule-based genetic algorithm for multi-objective job shop scheduling using fuzzy satisfaction levels, Computers \& Industrial Engineering, Vol. 86, 29-42, doi:10.1016/j.cie.2014.12.001

[19] Wang, L.; Ng, A. H. C. \& Deb, K. (2011). Multi-objective Evolutionary Optimisation for Product Design and Manufacturing, Springer, London, doi:10.1007/978-0-85729-652-8

[20] Zhang, Y.; Gong, D.-W. \& Ding, Z. (2012). A bare-bones multi-objective particle swarm optimization algorithm for environmental/economic dispatch, Information sciences, Vol. 192, 213-227, doi:10.1016/j.ins.2011.06.004

[21] Yin, Y.; Wang, D.-J.; Wu, C.-C. \& Cheng, T. C. E. (2016). CON/SLK due date assignment and scheduling on a single machine with two agents, Naval Research Logistics, Vol. 63, No. 5, 416-429, doi:10.1002/nav.21700

[22] Zhang, Y. Q. \& Zhang, H. (2020). Dynamic scheduling of blocking flow-shop based on multi-population aco algorithm, International Journal of Simulation Modelling, Vol. 19, No. 3, 529-539, doi:10.2507/ijsimm19-3-co15 